\title{
The application of the strength reduction shortest path method to the stability analysis of shallow buried tunnel
}

\section{Wei Wang}

central south university

Jiaqi Zhang ( $\nabla$ 895428190@qq.com )

central south university

Ao Liu

central south university

\section{Research Article}

Keywords: shortest path of strength reduction, limit equilibrium method, shallow tunnel, safety factor

Posted Date: June 28th, 2021

DOI: https://doi.org/10.21203/rs.3.rs-193335/v1

License: (1) (i) This work is licensed under a Creative Commons Attribution 4.0 International License.

Read Full License

Version of Record: A version of this preprint was published at Geotechnical and Geological Engineering on July 26th, 2021. See the published version at https://doi.org/10.1007/s10706-021-01944-3. 


\title{
The application of the strength reduction shortest path method to
}

\section{the stability analysis of shallow buried tunnel}

\author{
Wei Wang ${ }^{1}$, Jiaqi Zhang ${ }^{1}$, Ao Liu ${ }^{1}$ \\ ('School of Civil Engineering, Central South University, Hunan 410075, China)
}

\begin{abstract}
In order to improve the theoretical analysis of surrounding rock stability of shallow buried tunnel. The strength reduction shortest path theory is applied to the stability analysis of shallow buried tunnel surrounding rock, combined with the ultimate equilibrium strength reduction theory. We discussed the influence of the depth and span ratio of tunnel, cohesion, and internal friction angle on the shortest path of the strength reduction, and studied the effects of various factors on shallow buried tunnel safety relationship by using strength reduction factor of safety and the shortest path of the shallow buried tunnel surrounding rock and the grey relational analysis theory. The results show that: In the analysis of shallow buried tunnel in strength reduction, the approximate distribution obeys parabolic between reduction path length and the reduction ratio. When the strength reduction of cohesion is the shortest path the reduction rate is greater than the internal friction angle. The internal friction angle and cohesive force have a great influence on the stability of shallow tunnel under the method of shortest path of strength reduction. Finally, the comprehensive safety factor of shallow buried tunnel calculated by the finite element strength reduction shortest path method is greater than that calculated by the limit equilibrium method.
\end{abstract}

Key words : shortest path of strength reduction; limit equilibrium method; shallow tunnel; safety factor 


\section{Introduction}

The stability analysis of a shallow buried tunnel is an important theoretical basis to guide practical engineering. According to relevant statistics, some analysis methods proposed at present mainly include the limit equilibrium method, slip line method, limit analysis method, and finite element method. The limit analysis and finite element analysis are the research hotspots at present $[1-3]$

These two methods are mainly based on the strength reduction theory ${ }^{[4-6]}$, and the ultimate equilibrium state of the tunnel is achieved by reducing the shear strength parameters of rock-soil mass ${ }^{[7]}$. The traditional strength reduction method generally uses the same reduction coefficient for $c$ and $\tan \varphi$ of the shear parameters of rock and soil mass ${ }^{[8-9]}$. However, Fen Tang and Yingren Zheng et $a l^{[10-11]}$ believe that when the rock mass is damaged by sliding, the cohesion and internal friction angle of the soil mass play different roles, order, and degree.

On the other hand, the attenuation degree and speed of cohesive force $c$ and internal friction angle $\varphi$ are also different, and different reduction coefficients can accurately reflect the respective safety reserves of $c$ and $\varphi$, and the concept of double reduction method is proposed accordingly. Yuan ${ }^{[12]}$, Bai ${ }^{[13]}$, Zhao ${ }^{[14]}$, etc. also proposed their own strength double reduction methods in the slope stability analysis. Bai Bing et al ${ }^{[15]}$ pointed out that there should be an infinite number of reduction paths for double strength reduction, but ISAKOV ${ }^{[16]}$ believed that among all the reduction paths, there should be a path $L_{\min }$ to make the slope from the initial state to the limit state fastest, that is, the shortest path of strength reduction. Yuan Wei et al ${ }^{[17]}$ also believed that in the slope stability analysis, cohesion and internal friction angle should be reduced along the path with the shortest distance from the $c$ - $\tan \varphi$ critical curve in the process of attenuation failure.

At the same time, considering the complexity of soil parameters, the precision of the strength reduction method is affected by many factors. For this reason, Reginald et al ${ }^{[18]}$ compared and analyzed the strength reduction method with the limit equilibrium method, and proved that Poisson's ratio and Young's modulus had little influence on slope stability. Jing Zhoubao ${ }^{[19]}$ used the $A B A Q U S$ strength reduction method to study and showed that the cohesion and internal friction angle have significant effects on slope stability, the dilatancy angle has relatively little effect, and the elastic modulus and Poisson's ratio have almost no effect. Liu S Y et al ${ }^{[20]}$ analyzed the influence of dilatancy angle and Poisson's ratio on the safety coefficient of the slope by strength reduction 
method and found that the influence of dilatancy angle and Poisson's ratio on the safety coefficient of the slope was small. However, at present, the strength reduction shortest path method is mainly used in the slope ${ }^{[21-22]}$, and there are little researches on the tunnel.

Based on this, the strength reduction shortest path method is applied to the finite element strength reduction and ultimate equilibrium strength reduction analysis of shallow buried tunnels. The relationship between the length of the strength reduction path and the reduction ratio and the reasonable strength reduction ratio between the cohesion and the angle of internal friction under different parameters are discussed. The influence laws of buried depth and hole span ratio $H / D$, cohesion force $c$, and internal friction angle $\varphi$ on the shortest path of strength reduction were studied. Finally, the relationship between the reduction path, the safety coefficient of surrounding rock, and the grey correlation analysis theory are used to study the influence of various factors on the safety of the shallow buried tunnel. The research results enrich the analysis theory of surrounding rock stability of shallow-buried tunnel and provide some guidance for the construction of the shallowburied tunnel.

\section{Strength reduction shortest path theory}

The strength reduction shortest path theory ${ }^{[23-24]}$ assumes that the cohesion and internal friction angle of rock and soil mass is reduced by different proportions. The reduction coefficient of cohesion is $F_{c}$, and the reduction coefficient of internal friction angle is $F_{\varphi}$, the formula is as follows:

$$
\begin{aligned}
F_{C} & =\frac{c_{0}}{c_{S R T}} \\
F_{\varphi} & =\frac{\tan \varphi_{0}}{\tan \varphi_{S R T}}
\end{aligned}
$$

Where, $c_{0}$ and $\varphi_{0}$ are the initial cohesion and internal friction angle, $c_{S R T}$ and $\varphi_{S R T}$ are the reduced cohesion and internal friction angle.

As shown in Figure 1, assuming that the abscissa is $1 / F_{c}$ and the ordinate is $1 / F_{\varphi}$, the initial state $M_{0}$ of the tunnel and the line connecting the limit state line are reduced paths. There are countless strength reduction paths, and each reduction path is $L_{1} \cdots L_{n}$. There is a reduction path $L_{k}$, the intersection point $M_{k}$ of which is the limit state line. Under this reduction path, the cohesion and the angle of internal friction adopt the same reduction coefficient. In this case, the reduction path is the traditional strength reduction path. Among all the reduced paths, there should be a path 
$L_{\min }$ that causes the tunnel to reach the limit state at the earliest from the initial state. This path is the shortest reduced strength path.

The limit states under different reduction paths can be combined to obtain the limit state line shown in Figure 1, which can be expressed as a function of $F_{\varphi}=f\left(F_{c}\right)$. At the same time, considering the uncertainty of the reduction path, we cannot make a quantitative analysis of it. At this time, the reduction ratio $\lambda=\frac{F_{c}}{F_{\varphi}}$ is introduced, so that different strength reduction paths have a specific reduction ratio $\lambda$ corresponding to it. At this time, we can artificially assume different reduction ratios $\lambda$ to make the rock and soil mass be reduced according to different reduction paths, to quantitatively analyze the stability of the surrounding rock of the shallow buried tunnel.

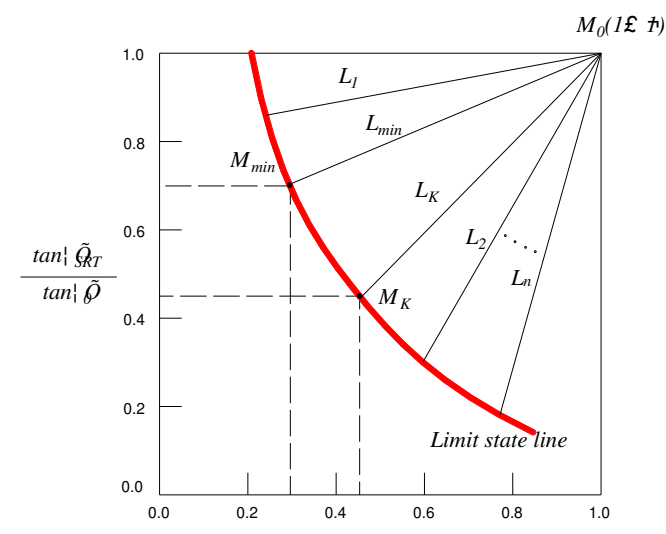

Fig.1 Schematic diagram of the shortest path of strength reduction

From Figure 1, it is easy to get the reduced path length from the initial state to the limit state of the tunnel under different reduced paths, the expression can be expressed as:

$$
L=\sqrt{\left(1-\frac{1}{F_{c}}\right)^{2}+\left(1-\frac{1}{F_{\varphi}}\right)^{2}}
$$

The area outside the limit state line near the origin is the instability zone, while the area inside the limit state line near the initial state of the tunnel is the safety zone. At this time, according to the path length distribution, a functional expression of the strength reduction path length and the tunnel comprehensive safety factor can be established, as in equation (4). When the strength reduction path takes the smallest value $L_{\min }$, the minimum comprehensive safety factor can be obtained ${ }^{[8]}$.

$$
F_{S}=\frac{1}{1-\frac{L}{\sqrt{2}}}
$$




\section{Finite element strength reduction shortest path analysis}

According to the existing mechanism of strength reduction, its principle is to gradually reduce the $c$ and $\varphi$ of rock and soil mass ${ }^{[25-26]}$. Regarding the non-proportional reduction of the cohesive force $c$ and the internal friction angle $\varphi$ of the rock and soil, we can specify the magnitude of the ABAQUS midfield variable, and then determine the limit state of the tunnel surrounding rock according to the instability criterion. Finally, the safety coefficient of the surrounding rock of the tunnel is obtained by using the relation between the length of the strength reduction path and the comprehensive safety coefficient of the tunnel. As for the instability criterion of rock and soil mass, the commonly used methods at present mainly include non-convergence criterion, plastic zone penetration criterion, and displacement mutation criterion ${ }^{[27-28]}$. In this paper, the sudden change in the settlement of the tunnel vault is selected as the criterion for the failure of the tunnel, and the safety factor at this time is selected for calculation and analysis.

\subsection{Finite element example analysis}

For a shallow tunnel, the ratio of the initial buried depth $H$ to the span $D$ is 1 , and both are $12 m$, the tunnel height is $9.72 \mathrm{~m}$, the surrounding rock mass density is $18 \mathrm{kN} / \mathrm{m}^{3}$, the elastic modulus is 1.3Gpa, and the Poisson's ratio is 0.35 . In the initial state, the cohesive force $c_{0}=120 \mathrm{kPa}$, and the internal friction angle is $\varphi_{0}=27^{\circ}$. As shown in Figure. 2, the overall model length $\times$ depth $=120 \mathrm{~m} \times 80 \mathrm{~m}$, and the tunnel height $\times$ width $=9.72 \mathrm{~m} \times 12 \mathrm{~m}$. The Mohr-Coulomb constitutive model is used in the calculation. The nodes at the bottom of the model limit the displacement in the $X$ and $Y$ directions, and the nodes on both sides limit the displacement in the $X$-direction.

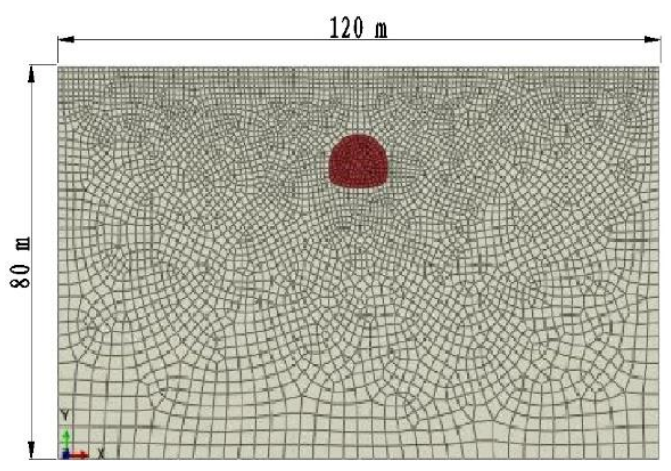

(a) Overall model size 


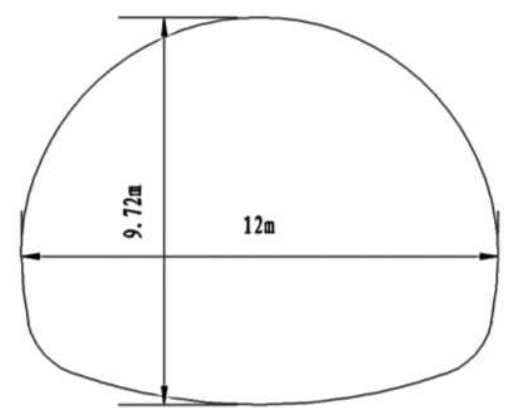

(b) Tunnel size

Fig.2 Finite element calculation model

In order to make the distribution of strength reduction path more uniform as far as possible, this paper assumes that the value range of reduction ratio is $0.5 \sim 2.0$, to realize the reduction of rocksoil mass parameters according to different reduction paths. Combining the results of finite element limit calculation and instability criterion, the strength reduction path length (expressed as the square of the length) under different reduction ratios can be obtained as shown in Figure 3. With the increase of the reduction ratio, the length of the reduction path first decreases and then increases, and the minimum value occurs when $\lambda=1.5$. At this time, the corresponding reduction path $L_{m i n}$ is the shortest path mentioned above, and the comprehensive safety factor of the tunnel is 1.5. By fitting the relevant data, the distribution between the reduction ratio and the path length is approximated by a parabola.

Figure 4 shows the distribution of the plastic zone obtained by reducing the strength of the rock mass extracted in the finite element calculation according to the reduction ratio $\lambda=1.5$. By connecting the points with the largest plastic deformation of the surrounding rock into a line, the potential failure surface of the surrounding rock can be approximated.

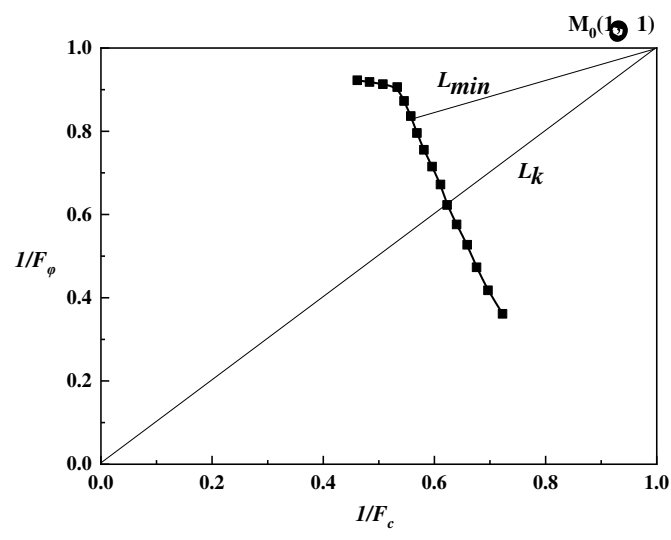

(a) Shortest path diagram of strength reduction

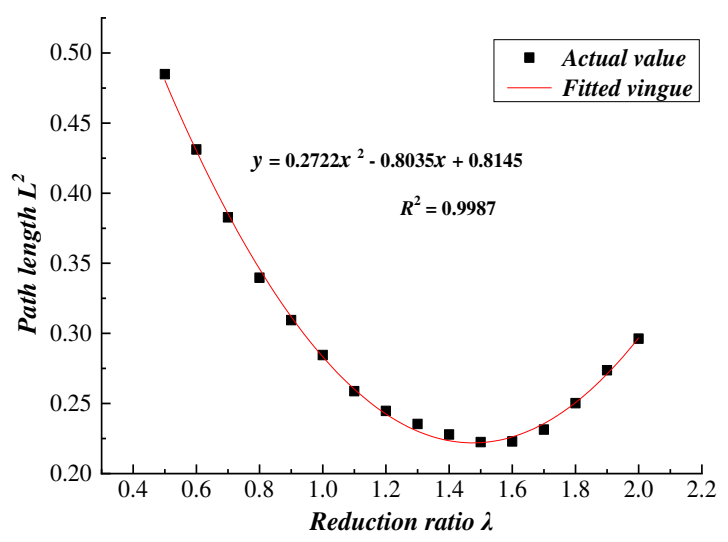

(b) Path length change diagram

Fig.3 Path length under different reduction factor 


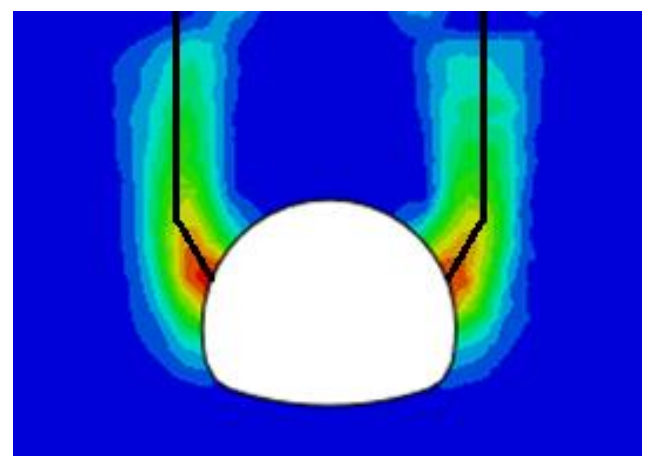

Fig.4 Failure modes under different reduction factor

\subsection{Effects of different parameters on tunnel strength reduction shortest path}

Previous studies have shown that elastic modulus, dilation angle, and Poisson's ratio have little influence on rock mass stability ${ }^{[29-31]}$. This paper mainly studies the influence law of buried depth and hole span ratio $H / D$, cohesion $c$, and internal friction angle $\varphi$ on the shortest path of finite element strength reduction.

Buried depth and hole span ratio $(H / D)$ select five working conditions of $0.5,0.75,1.0,1.25$, and 1.5. Cohesion $c_{0}$ selects five working conditions of $80,100,120,140$, and $160 \mathrm{kPa}$. Five operating conditions of $17^{\circ}, 19.5^{\circ}, 22^{\circ}, 24.5^{\circ}$, and $27^{\circ}$ are selected for the initial internal friction angle $\varphi_{0}$. The specific calculation conditions are shown in Table 1, and the values of other parameters of all operating conditions remain unchanged.

Table 1. Calculation condition

\begin{tabular}{cccc}
\hline$H / D$ & $C 0(\mathrm{kPa})$ & $\varphi_{0}\left({ }^{\circ}\right)$ \\
\hline $0.5,0.75,1.0,1.25,1.5$ & 120 & 22 \\
\hline 1.0 & $80,100,120,140,160$ & 22 \\
\hline 1.0 & 120 & $17,19.5,22,24.5,27$ \\
\hline
\end{tabular}

Figures. 5-7 show the relationship curves of the path length with the reduction ratio under different $H / D$, cohesion $c_{0}$, and internal friction angle $\varphi_{0}$. It can be seen from the figure that the square of the length $L^{2}$ of the strength reduction path and the reduction ratio $\lambda$ approximately obey the parabolic distribution law.

It can be seen from Fig. 5 that as $H / D$ increases, the reduced shortest path $L_{\min }^{2}$ gradually decreases, and the corresponding reduction ratio $\lambda$ gradually decreases. 
Table 2. Variation of reduction ratio under different $H / D$

\begin{tabular}{cc}
\hline$H / D$ & $\lambda$ \\
\hline 0.5 & 1.7 \\
0.75 & 1.6 \\
1.0 & 1.5 \\
1.25 & 1.4 \\
1.5 & 1.3 \\
\hline
\end{tabular}

When $H / D$ is larger, the reduction ratio $\lambda$ is closer to 1 , that's to say, as the $H / D$ increases, the shortest path of strength reduction is closer to the traditional strength reduction path.

It can be seen from Figure 6 that as the initial cohesion $c_{0}$ increases, the reduced shortest path $L_{\text {min }}^{2}$ gradually increases, and the corresponding reduction ratio $\lambda$ becomes larger.

Table 3. Variation of reduction ratio under different cohesion $c_{0}$

\begin{tabular}{cc}
\hline$c_{0} / \mathrm{kPa}$ & $\lambda$ \\
\hline 80 & 1.1 \\
100 & 1.3 \\
120 & 1.5 \\
140 & 1.7 \\
160 & 2.0
\end{tabular}

It can be seen from Figure 7 that when the initial internal friction angle increases, the shortest

171 path length $L_{\min }^{2}$ for strength reduction gradually increases, while the reduction ratio $\lambda$ at this time 172 remains unchanged.

Table 4. Variation of reduction ratio under different internal friction angle $\varphi_{0}$

\begin{tabular}{cc}
\hline$\varphi_{0}$ & $\lambda$ \\
\hline $17^{\circ}$ & 1.5 \\
$19.5^{\circ}$ & 1.5 \\
$22^{\circ}$ & 1.5 \\
$24.5^{\circ}$ & 1.5 \\
$27^{\circ}$ & 1.5
\end{tabular}

174 This means that the change of the internal friction angle will not affect the reduced path of the 175 shortest path of strength reduction. 
176

177

178

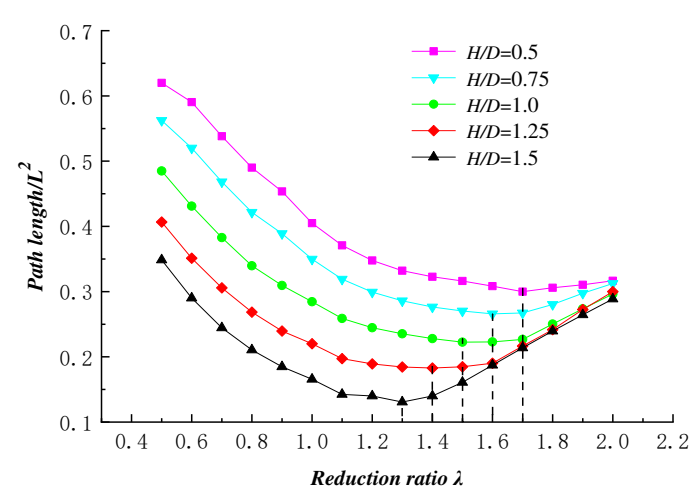

Fig.5 Reduction factor and path length under different $H / D$

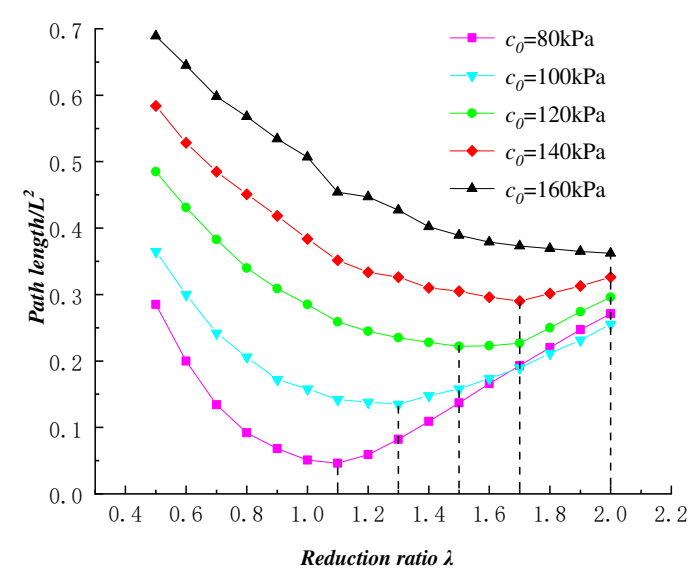

Fig.6 Reduction factor and path length under different $c_{0}$

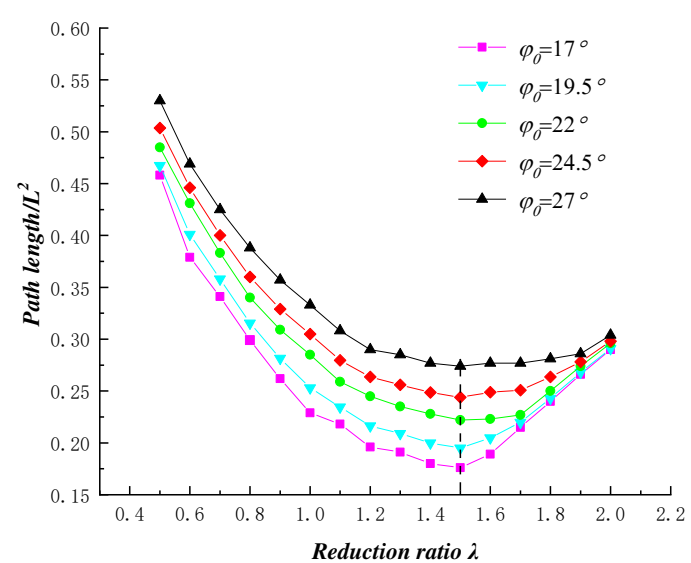

Fig.7 Reduction factor and path length under different $\varphi_{0}$

Through statistics of the reduction ratios under the shortest path of strength reduction, the reduction ratios under the shortest path of strength reduction are all greater than 1 . This shows that when the rock and soil reach the limit state according to the shortest reduction path, the reduction of cohesion is greater than the reduction of the internal friction angle. 


\section{Analysis of ultimate equilibrium strength reduction shortest path method}

\subsection{Limit equilibrium analysis model}

According to Terzaghi's theory and the potential fracture surface in Figure 4, the ultimate equilibrium failure mode of shallow buried tunnels is obtained. As shown in the figure, the potential fracture surfaces are $A B C$ and $A_{l} B_{l} C_{l}$, and the following assumptions are made:

(1) The surrounding rock near the shallow buried tunnel slides along potential fracture planes $A B$ and $A_{l} B_{1}$, and the sliding plane forms an angle $\alpha\left(\alpha=45^{\circ}-\varphi / 2\right)$ to the vertical, and $\varphi$ is the initial internal friction angle;

(2) The fracture surface $B C$ and $B_{l} C_{l}$ are vertical to the ground surface, $B D B_{l}$ is parallel to the ground surface, and $D$ is located at the vault.

Considering the symmetry, only the limit equilibrium analysis of the sliding body $A B D$ can be carried out, and its safety factor $K$ can be expressed as:

$$
K=\frac{F_{R}}{F_{T}}=\frac{T+T_{x} \sin \alpha}{\left(p a_{1}+W\right) \cos \alpha}
$$

In the formula: $F_{R}$ and $F_{T}$ respectively represent the anti-skid force and the sliding force.

According to the static balance conditions in the horizontal direction:

$$
N \cos \alpha=T \sin \alpha+T_{x}
$$

Apply the Moore Coulomb strength criterion to the potential failure surface $A B$ :

$$
T=\frac{c h}{\cos \alpha}+N \tan \varphi
$$

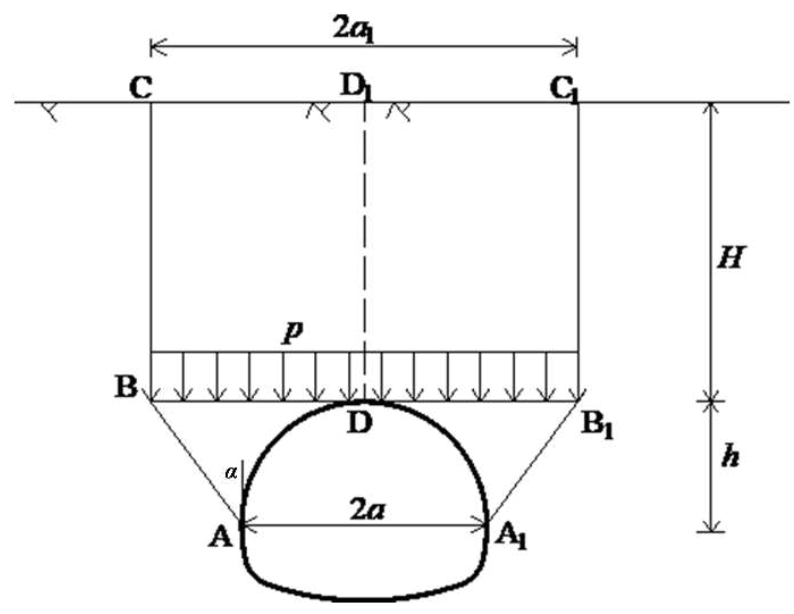

(a) 
206

207

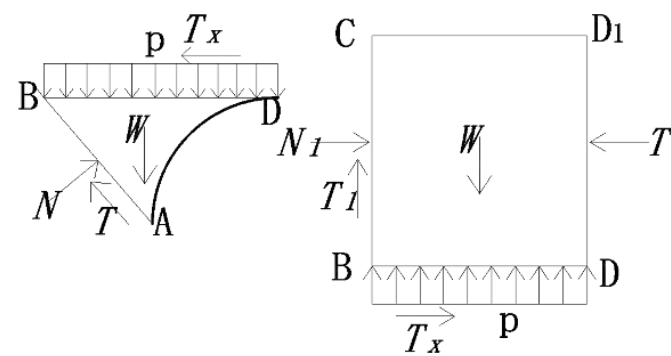

(b)

Fig.8 Stress analysis diagram of limit equilibrium state

Substituting equation (7) into equation (6) can get:

$$
N=\frac{\operatorname{chtan} \alpha+T_{x}}{\cos \alpha-\sin \alpha \tan \varphi}
$$

Considering that $\mathrm{BD}$ is not a sliding surface, therefore:

$$
T \approx c a_{1}
$$

Suppose $p=\gamma H, \mathrm{H}$ is the buried depth of the tunnel, $\mathrm{p}$ is the surrounding rock pressure of a shallow tunnel, and $\alpha=45^{\circ}-\varphi / 2$.

From the above formula, the formula (4) is finally transformed into:

$$
\frac{K}{c}=\frac{15.93+17.47 \tan \varphi /(0.819-0.574 \tan \varphi)}{183.6 H+365.85}
$$

When $\mathrm{K}=1.0$, the shallow tunnel is in a state of limit equilibrium.

Suppose $\lambda=\frac{F_{c}}{F_{\varphi}}, F_{c}=\frac{c_{0}}{c}, F_{\varphi}=\frac{\tan \varphi_{0}}{\tan \varphi}$ the formula (10) can be obtained:

$$
\lambda=\frac{17.47 \mathrm{c}_{0} \tan \varphi_{0} /\left(0.819 \mathrm{~F}_{\varphi}^{2}-0.574 \mathrm{~F}_{\varphi} \tan \varphi_{0}\right)}{183.6 \mathrm{H}+365.85}+\frac{\frac{15.93 \mathrm{c}_{0}}{\mathrm{~F}_{\varphi}}}{183.6 \mathrm{H}+365.85}
$$

\subsection{The influence of different parameters on the shortest path of strength reduction of shallow buried tunnel}

The working conditions in Table 1 are still used for calculation, and the influence of the three major factors of buried depth and hole-span ratio, cohesion, and internal friction angle on the shortest path of ultimate equilibrium strength reduction is obtained.

Through comparison, it can be found that the calculation results of the limit equilibrium method are basically consistent with the finite element numerical calculation results:

(1) As $\mathrm{H} / \mathrm{D}$ increases, the shortest path $L_{\min }$ of strength reduction gradually decreases, and the corresponding reduction ratio $\lambda$ gradually decreases. 
(2) With the increase of initial cohesion force $c_{0}$, the reduction shortest path $L_{\min }$ gradually 231 increases, and the corresponding reduction ratio $\lambda$ also gradually increases.

(3) With the increase of the initial internal friction angle, the reduced shortest path $L_{\text {min }}$ gradually increases, and the corresponding reduction ratio $\lambda$ remains unchanged.

234

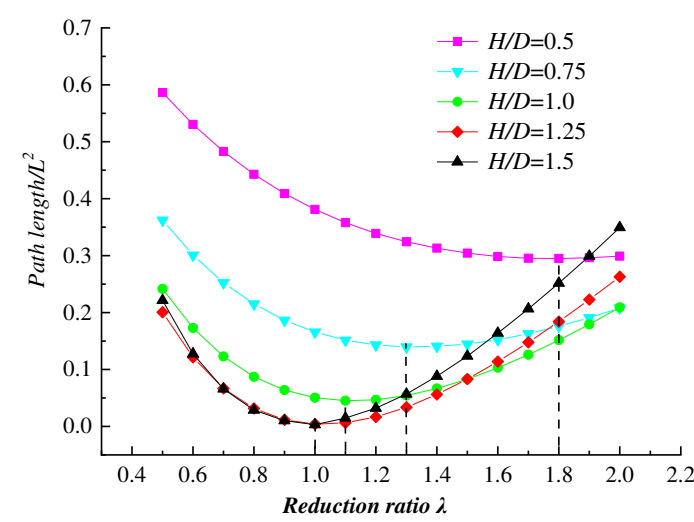

Fig.9 Reduction factor and path length under different $H / D$

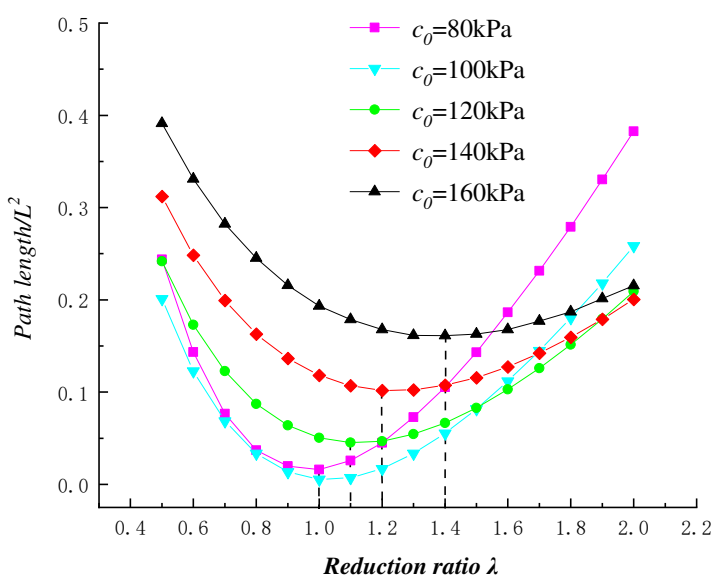

Fig.10 Reduction factor and path length under different $c_{0}$

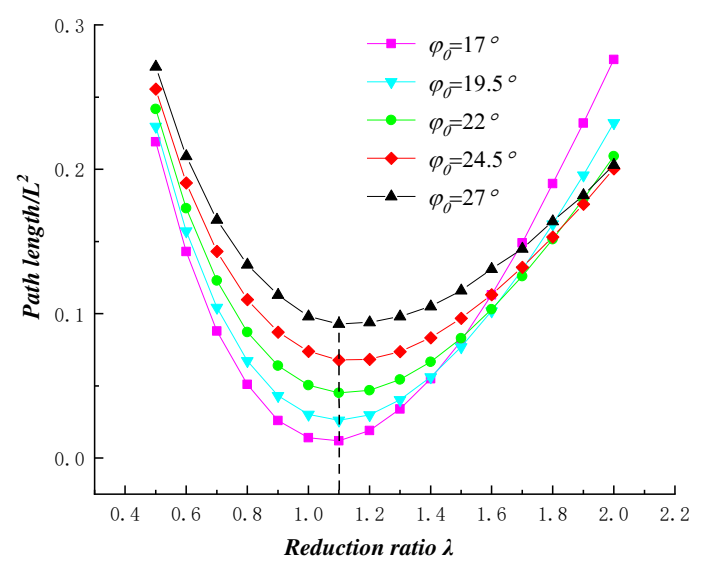

$12 / 19$ 
4 Comparison of safety factors and sensitivity analysis of influencing factors under different methods

\subsection{Comparative analysis of comprehensive safety factors under different methods}

It can be seen from Equation (4) that the length of the shortest path by strength reduction can indirectly represent the safety factor. To further reflect the difference and relation between the shortest path by strength reduction by finite element method and the shortest path by ultimate equilibrium strength reduction, the comprehensive safety factor of the two different methods under different parameters is obtained as shown in Figure 12 14 . the tunnel in the initial state, the greater the overall safety factor, and the two are approximately in a linear relationship. When the buried depth and span ratio are small, the safety factors obtained under the finite element method and the limit equilibrium method are relatively close. However, the finite element method of safety coefficient calculated under different parameters is larger than the limit equilibrium method, which indicates that shallow-buried tunnels are more likely to be damaged under the limit equilibrium method. This is because in the calculation of the limit equilibrium method, the value of the surrounding rock pressure $P$ is larger, and the actual surrounding rock pressure $P$ is smaller than $\gamma h$, but in general, the two laws are consistent.

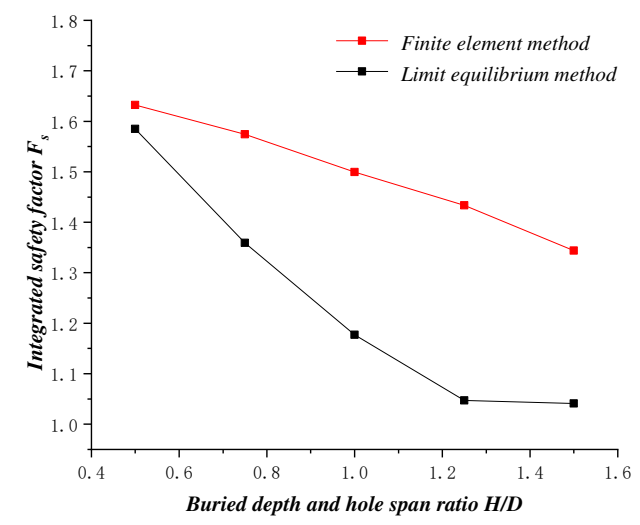

Fig.12 The relationship curves between the safety factor and $H / D$ under different method 


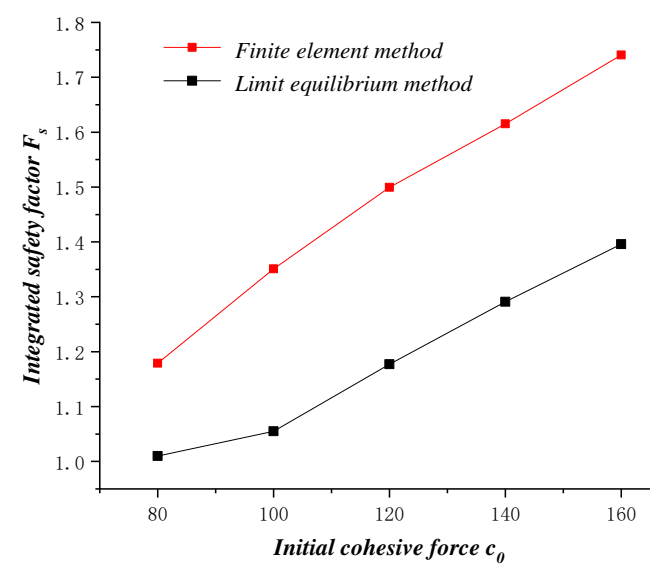

Fig.13 The relationship curves between the safety factor and $c o$ under different method

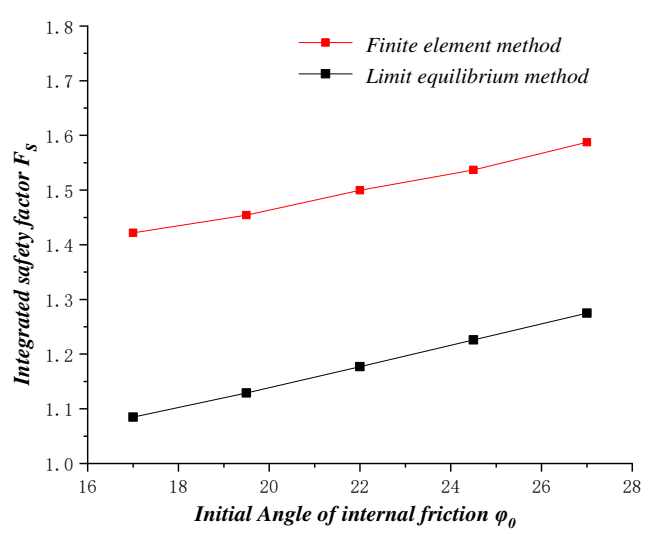

Fig.14 The relationship curves between the safety factor and $\varphi_{0}$ under different method

Table 5. Safety factor under different $H / D$

\begin{tabular}{cccccc}
\hline$H / D$ & 0.5 & 0.75 & 1.0 & 1.25 & 1.5 \\
\hline$F S$ & 1.632 & 1.574 & 1.500 & 1.434 & 1.344 \\
\hline
\end{tabular}


Table 6. Safety factor under different $c_{0}$

\begin{tabular}{cccccc}
\hline$c$ & 80 & 100 & 120 & 140 & 160 \\
\hline$F S$ & 1.179 & 1.351 & 1.500 & 1.615 & 1.740 \\
\hline
\end{tabular}

Table 7. Safety factor under different $\varphi 0$

\begin{tabular}{cccccc}
\hline$\varphi$ & 17 & 19.5 & 22 & 24.5 & 27 \\
\hline$F S$ & 1.422 & 1.454 & 1.500 & 1.537 & 1.588 \\
\hline
\end{tabular}

According to different influencing factors and working conditions, the initial matrix of gray correlation calculation is obtained as follows:

$$
X=\left[\begin{array}{l}
X_{1} \\
X_{2} \\
X_{3}
\end{array}\right]=\left[\begin{array}{ccccc}
0.5 & 0.75 & 1.0 & 1.25 & 1.5 \\
80 & 100 & 120 & 140 & 160 \\
17 & 19.5 & 22 & 24.5 & 27
\end{array}\right]
$$

1) Perform averaging on the above $X$ and $Y$ matrix sequence, let: and minimum values:

$$
\Delta_{\max }=\max \left(\Delta_{i j}\right) \Delta_{\min }=\min \left(\Delta_{i j}\right)
$$

3) Find the gray correlation coefficient matrix

Before the analysis, the distance between the comparison point and the reference point should be sorted out, and the overall analysis should be used to calculate the difference and correlation between the factors. Finally, the correlation coefficient between the comparison factor and the reference factor is obtained through formula (15), which reflects its correlation.

$$
\gamma_{i j}=\frac{\Delta_{\min }+\xi \Delta_{\max }}{\Delta_{i j}+\xi \Delta_{\max }}
$$

In the formula: $\zeta$ is the resolution coefficient, which is generally 0.5 .

4) Find the degree of grey correlation 
relevant coefficient, and the relevance comparison of the impact size is made accordingly.

$$
A_{i}=\frac{1}{n} \sum_{j=1}^{n} \gamma_{i j}
$$

Using the above formula to calculate, the gray correlation degrees of $H / D$, cohesion $c$, and internal friction angle $\varphi$ of the shallow tunnel are $0.5347,0.7805$, and 0.7661 , respectively. Therefore, in the process of calculating the stability of shallow tunnels by the strength reduction shortest path method, the internal friction angle $\varphi$ and cohesive force $\mathrm{c}$ have a greater impact on the safety of shallow tunnels.

303

\section{Conclusion}

(1) In shallow tunnels, the reduced path length (expressed by the square of the length) obtained by the finite element strength reduction method and the ultimate equilibrium strength reduction method and the reduction ratio approximately obey the parabolic distribution law.

(2) The strength reduction shortest path $L_{\min }$ and the corresponding reduction ratio $\lambda$ gradually decrease with the increase of $\mathrm{H} / \mathrm{D}$, and gradually increase with the increase of the initial cohesive force $\mathrm{c} 0$; with the increase of the initial internal friction angle, the reduction is the shortest The path $L_{m i n}$ gradually increases, and the corresponding reduction ratio $\lambda$ remains unchanged.

(3) The comprehensive safety factor of shallow tunnel calculated by the finite element strength reduction shortest path method is greater than the comprehensive safety factor calculated by the ultimate equilibrium strength reduction shortest path method. The strength reduction shortest path method can be a new method to analyze the stability of shallow tunnels.

(4) Among the factors affecting the stability of shallow tunnels calculated by the strength reduction shortest path method, the internal friction angle $\varphi$, and cohesion $\mathrm{c}$ have a greater impact on the safety of shallow tunnels.

\section{Conflict of interest statement}

We declare that we have no financial and personal relationships with other people or organizations that can inappropriately influence our work, there is no professional or other personal interest of any nature or kind in any product, service or company that could be construed as influencing the position presented in, or the review of, the manuscript entitled, "The application of the strength reduction shortest path method to the stability analysis of shallow buried tunnel". 


\section{References}

325 [1] Wenxi Fu, Yi Liao. Non-linear shear strength reduction technique in slope stability calculation [J]. Computers and Geotechnics, 2010, 37(3): 288-298.

[2] Yiliang Tu, Xinrong Liu, Zuliang Zhong, Yayong Li. New criteria for defining slope failure using the strength reduction method [J]. Engineering Geology, 2016, (212): 63-71.

[3] Qing-qing JIANG. Strength reduction method for slope based on a ubiquitous-joint criterion and its application [J]. Mining Science and Technology (China), 2009, 19(4): 452-456.

[4] Zienkiewicz O C, Humpeson C, Lewis R W. Associated and nonassociated visco-plasiticity in soil mechanics [J]. Geotechnique, 1975, 25(4): 671-689.

[5] Haibin Wang, Yongtao Yang, Guanhua Sun, Hong Zheng. A stability analysis of rock slopes using a nonlinear strength reduction numerical manifold method [J]. Computers and Geotechnics, 2021, (129): 103864.

[6] Guanhua Sun, Shan Lin, Hong Zheng, Yunzhi Tan, Tan Sui. The virtual element method strength reduction technique for the stability analysis of stony soil slopes [J]. Computers and Geotechnics, 2020, (119): 103349.

[7] F. Tschuchnigg, H.F. Schweiger, S.W. Sloan. Slope stability analysis by means of finite element limit analysis and finite element strength reduction techniques. Part II: Back analyses of a case history [J]. Computers and Geotechnics, 2015, (70): 178-189.

[8] AN Yonglin, HUANG Kan, PENG Limin, et al. Analysis of overall stability of tunnel by strength reduction method [J]. Highway Traffic Science and Technology, 2011, 28(4): 91-95.

[9] HE Xinliang. Study on self stability of tunnel surrounding rock based on strength reduction method [D]. Hunan University, 2013.

[10] TANG Fen, ZHENG Ying-ren, ZHAO Shang-yi. Discussion on two safety factors for progressive failure of soil slope[J]. Chinese Journal of Rock Mechanics and Engineering, 2007, 26(7): 1402-1407.

[11] TANG Fen, ZHENG Ying-ren. Mechanism analysis on dual reduction factors about the progressive failure of slope [J]. Chinese Journal of Underground Space and Engineering, 2008, 4(3): 436-441.

[12] Yuan Wei, Bai Bing, Li Xiaochun, et al. A Strength reduction method based on double reduction parameters and its application [J]. Journal of Central South University, 2013, 20(9): 2555-2562.

350 [13] Bai bing, Yuan Wei, Li Xiaochun. A new double reduction method for slope stability analysis [J]. Journal of Central South University, 2014, 21(3): 1158-1164.

[14] Lianheng Zhao, Feng Yang, Yingbin Zhang, etc. Effects of shear strength reduction strategies on safety factor of homogeneous slope based on a general nonlinear failure criterion [J]. Computers and Geotechnics, 2015, (63): $215-228$.

[15] Bai Bing, Yuan Wei, Shi Lu, et al. A kind of double reduction method and classical strength reduction method 
[16] Isakov A, Moryachkov Y. Estimation of Slope Stability Using Two-Parameter Criterion of Stability [J]. International Journal of Geomechanics, 2014, 14(3): 613-624.

[17] LI Xiaochun, WANG Wei, et al. Study on strength reduction method based on double reduction parameters [J]. Rock and Soil Mechanics, 2016, 37(8): 2222-2230.

[18] Reginald Hammah, Thamer Yacoub, Brent Corkum, etc. A comparison of finite element slope stability analysis with conventional limit-equilibrium investigation [J]. 2005

[19] JING Zhoubao. Analysis of influencing factors of slope stability based on ABAQUS strength decreasing algorithm [J]. Qinghai transportation technology, 2015, (2): 56-60.

[20] Liu S Y, Shao L T, Li H J. Slope stability analysis using the limit equilibrium method and two finite element methods [J]. Computers and Geotechnics, 2015, (63): 291-298.

[21] Oumaima Abra, Mahdi Ben Ftima. Development of a new design approach of reinforced concrete structures based on strength reduction method [J]. Engineering Structures, 2020, (207): 110192.

[22] Chaowei Sun, Junrui Chai, Zengguang Xu, Yuan Qin, Xingzhou Chen. Stability charts for rock mass slopes based on the Hoek-Brown strength reduction technique [J]. Engineering Geology, 2016, (214): 94-106.

[23] J.Z. Ma, J. Zhang, H.W. Huang, L.L. Zhang, J.S. Huang. Identification of representative slip surfaces for reliability analysis of soil slopes based on shear strength reduction [J]. Computers and Geotechnics, 2017, (85): 199-206.

[24] Shanbi Peng, Wen Liao, Enbin Liu. Pipe-soil interaction under the rainfall-induced instability of slope based on soil strength reduction method [J]. Energy Reports, 2020, (6): 1865-1875.

[25] Horn-Da Lin, Wei-Chien Wang, An-Jui Li. Investigation of dilatancy angle effects on slope stability using the 3D finite element method strength reduction technique [J]. Computers and Geotechnics, 2020, (118): 103295.

[26] Zhibao Nie, Zhihong Zhang, Hong Zheng. Slope stability analysis using convergent strength reduction method [J]. Engineering Analysis with Boundary Elements, 2019, (108): 402-410.

[27] Yuan Wei, Li Jiaxin, Li Zonghong, Wang Wei, Sun Xiaoyun. A strength reduction method based on the Generalized Hoek-Brown (GHB) criterion for rock slope stability analysis [J]. Computers and Geotechnics, 2020, (117): 103240.

[28] ZHANG Aijun, MO Haihong. Improving displacement mutation criterion of slope failure in strength reduction finite element method [J]. Rock and Soil Mechanics, 2013, (s2): 332-337.

[29] ZHOU Yuanfu, DENG Jianhui, CUI Yulong, et al. Instability criterion of three-dimensional slope based on strength reduction method [J]. Rock and Soil Mechanics, 2014, (5): 1430-1437.

[30] K. Ma, B. Feng, D.Y. Zhuang, X.F. Guo, Q. Gao. Distance effects of the fault on the surrounding rock mass stability of the main powerhouse at the Huanggou pumped-storage power station [J]. Tunnelling and Underground Space Technology, 2020, (106): 103568.

[31] Ke Ma, Jianhai Zhang, Zhong Zhou, Nuwen Xu. Comprehensive analysis of the surrounding rock mass stability 

Underground Space Technology, 2020, (104): 103525.

[32] LI Zhijia, ZHANG Dingli, FANG Qian, LI Shu. An Analysis on Factors Influencing tability of Soil Slope by Strength Reduction Method [J]. Tunnel Construction, 2013, (10):854-859. 